\title{
Stiction, adhesion energy, and the Casimir effect in micromechanical systems
}

\author{
E. Buks and M. L. Roukes \\ Condensed Matter Physics, California Institute of Technology, Pasadena, California 91125 \\ (Received 3 August 2000; revised manuscript received 5 October 2000; published 2 January 2001)
}

\begin{abstract}
We measure the adhesion energy of gold using a micromachined cantilever beam. Stress and stiffness of the beam are characterized by measuring the spectrum of mechanical vibrations and the deflection due to external force. We induce stiction between the beam and a nearby surface, employing capillary forces to determine the adhesion energy $\gamma$. The obtained value $\gamma=0.06 \mathrm{~J} / \mathrm{m}^{2}$ is a factor of 6 smaller than that predicted by idealized theory. This discrepancy may arise from surface roughness or an adsorbed layer intervening between the contacting surfaces in these mesoscopic structures.
\end{abstract}

DOI: 10.1103/PhysRevB.63.033402

PACS number(s): 68.03.Cd, 68.35.Gy, 87.80.Mj

The Casimir effect ${ }^{1}$ is one of the most striking consequences of quantum electrodynamics (for a recent review, see Ref. 2). The dependence of the ground state energy of the electromagnetic field upon boundary conditions gives rise to an observable force between macroscopic bodies. A significant enhancement in the accuracy of measuring the Casimir force has been achieved recently with experiments employing the torsion pendulum ${ }^{3}$ and atomic force microscope (AFM). ${ }^{4}$ Casimir effect investigations may open the way for experimental observation of new fundamental forces arising from the hypothetical extra dimensions predicted by modern theories (see, for example, Ref. 5). However, to enable such studies it is crucial to improve experimental techniques. The Casimir force, in addition to its fundamental interest, also plays an important role in the fabrication and operation of microelectromechanical systems (MEMS). This technology allows fabrication of a variety of on-chip fully integrated sensors and actuators with a rapidly growing number of applications. One of the principal causes of malfunctioning in MEMS is stiction, namely, the collapse of movable elements into nearby surfaces, resulting in their permanent adhesion (for a review, see Refs. 6 and 7). This can occur during fabrication, especially due to capillary forces present during drying of a liquid from the surface of the sample, or during operation. ${ }^{8}$ It was argued recently that the Casimir effect is often an important underlying mechanism causing this phenomenon. 9

Here we report our experimental study of surface-surface interactions using micromachined $\mathrm{Au}$ cantilevers. In particular, we focus upon the extreme manifestation of the Casimir interaction, namely, adhesion between surfaces and the associated energy of this process. Traditionally, adhesion energy was studied experimentally by applying external forces to bulk materials and measuring the resultant contraction and/or cleavage. ${ }^{10}$ For these experiments the adhesion is associated with the perfectly smooth and clean internal surfaces of the bulk material. On the other hand, in many practical applications and in particular for MEMS devices surfaces are not ideal. In the present experiment we study this case by measuring adhesion between surfaces that may have some roughness and/or adsorbed contamination. These imperfections may alter the surfaces' properties, thus leading to a modified adhesion energy.
The structures we use are designed to allow straightforward and unambiguous interpretation of our results. We use bulk micromachining (rather than surface micromachining), in which the substrate is completely removed beneath the sample. This greatly simplifies the boundary conditions of the electromagnetic field in the vicinity of the sample. Moreover, we avoid using multilayered structures, since their internal stresses generally play an important role and theoretical modeling is thus more difficult. We use metallic rather than semiconductor structures to minimize the possibility of parasitic bound surface charge attraction.

After characterizing the mechanical properties of the beam, we induce stiction between the beam and a nearby electrode. The shape of the beam after adhesion and the elastic energy associated with this configuration allow us to determine the attractive surface energy. Similar methods were employed to measure the adhesion energy of stress-free $\mathrm{Si}^{6,7}$ Note, however, that generally mechanical properties such as stress have to be characterized in order to accurately determine the elastic energy. We conclude by comparing our results with previous measurements and with theory.

The bulk micromachining process employed for sample fabrication is described in Fig. 1. In the first step chemical vapor deposition is employed to deposit a $70 \mathrm{~nm}$ thick layer of $\mathrm{Si}_{3} \mathrm{~N}_{4}$ on the front and back sides of a $\mathrm{Si}$ wafer. A square window is opened in the $\mathrm{Si}_{3} \mathrm{~N}_{4}$ on the back using photolithography and wet etching [Fig. 1(a)]. The high selectivity and anisotropic etching properties of $\mathrm{KOH}$ are employed to form the structure shown in Fig. 1(b), with a $300 \mu \mathrm{m}$ square of $\mathrm{Si}_{3} \mathrm{~N}_{4}$ suspended membrane on the front side of the wafer. The gold beam and nearby electrodes are fabricated on top of the membrane using $e$-beam lithography and thermal evaporation [Fig. 1(c)]. The beam has length $l=200 \mu \mathrm{m}$, width $a=0.24 \mu \mathrm{m}$, and thickness $t=0.25 \mu \mathrm{m}$ (measured using AFM). In the last step the membrane is removed using electron cyclotron resonance plasma etching with an $\mathrm{Ar} / \mathrm{NF}_{3}$ gas mixture bombarding the back side of the sample. This leaves the gold beam suspended [Fig. 1(d)]. Figure 1(e) is a micrograph showing a side view of the device.

To characterize the mechanical properties of the beam we employ two methods, namely, measurement of the resonance frequencies of the beam and measurement of the deflection due to an external force. Both methods lead to similar conclusions. 

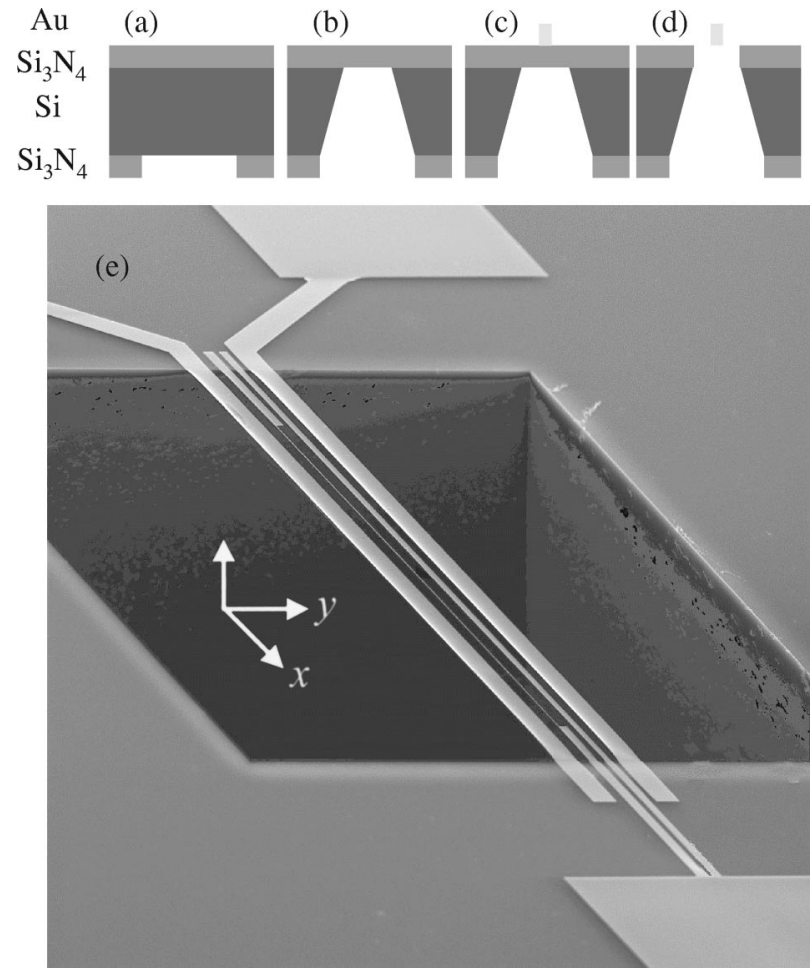

FIG. 1. The device is fabricated using bulk micromachining techniques. In steps (a) and (b) a suspended membrane of silicon nitride is formed. A gold beam is fabricated on top of the membrane (c) and the membrane is etched, leaving the beam suspended (d). Side view micrograph of the device is seen in (e).

The equation of motion of the beam is given by

$$
\frac{\partial^{2} y}{\partial x^{2}}-\zeta^{2} l^{2} \frac{\partial^{4} y}{\partial x^{4}}=(\rho A / T) \frac{\partial^{2} y}{\partial t^{2}}-f / T,
$$

where $\zeta^{2}=E A a^{2} / 12 T l^{2}$, with $E$ being Young's modulus, $A$ $=a t$ the area of the beam's cross section, $T$ the tension, $\rho$ the mass density, and $f$ the density of external force. ${ }^{11}$ The clamping of the beam on both sides is taken into account using the boundary conditions $y( \pm l / 2)=(\partial y / \partial x)( \pm l / 2)$ $=0$.

The dimensionless parameter $\zeta$ indicates the relative effect of stiffness compared with tension on the dynamics of the beam. As we shall see below, $\zeta \ll 1$ in our case; therefore we expand the resonance frequencies of the system in powers of $\zeta$ using perturbation theory. To second order we find

$$
\nu_{n}=n \nu_{0}\left[1+2 \zeta+\left(4+n^{2} \pi^{2} / 2\right) \zeta^{2}\right],
$$

where $\nu_{0}=\sqrt{T / \rho A} / 2 l$. The equally spaced spectrum obtained for the case $\zeta=0$ is the same as for a stiffness-free beam with boundary conditions $y( \pm l / 2)=0$. Note that the terms that make the spectrum unequally spaced are of order $O\left(\zeta^{2}\right)$.

The resonance frequencies are measured in situ using a commercial scanning electron microscope (SEM). The electron beam is focused on a point near the edge of the gold beam and the output signal from a photomultiplier (serving as a secondary electron detector) is monitored using a spectrum analyzer to detect mechanical displacement [see Fig.

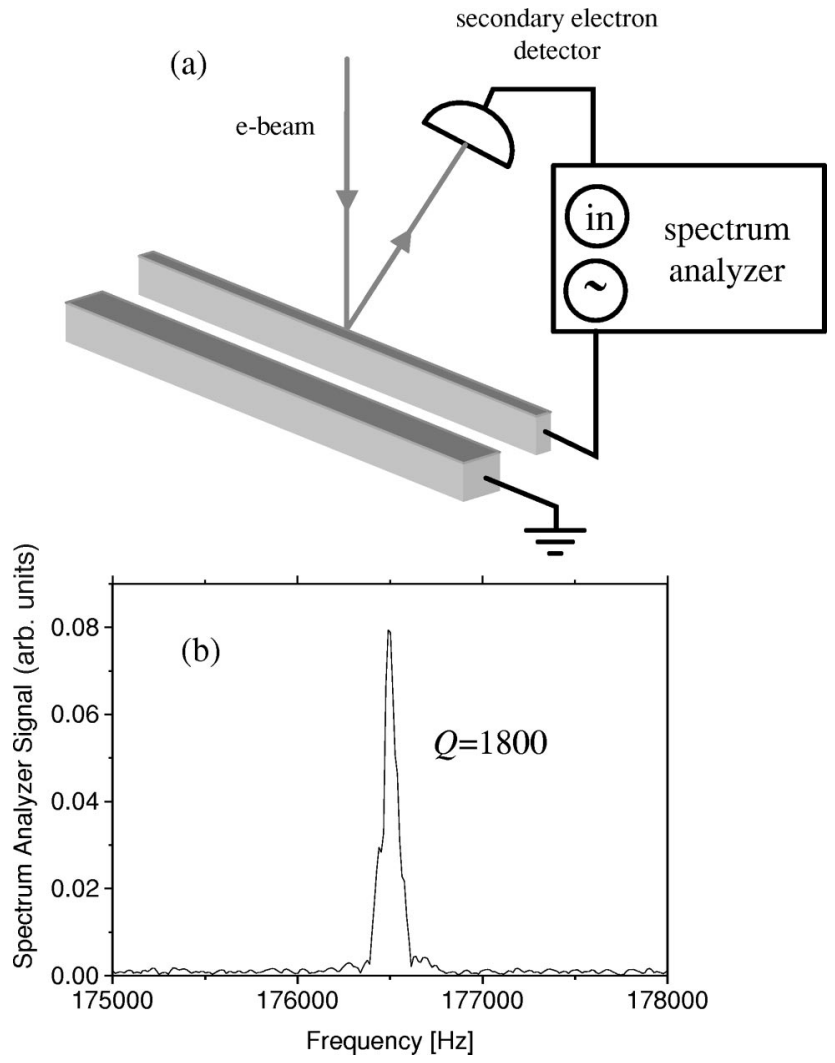

FIG. 2. (a) The setup employed to detect the resonance frequencies of the beam. (b) Peak in the displacement noise associated with thermal excitation of the fundamental mode.

2(a)]. Note that this detection scheme is sensitive almost exclusively to motion in the plane of the sample.

Without applying any external excitation we find a pronounced peak near $\nu_{1}=176.5 \mathrm{kHz}$ associated with thermal excitation of the fundamental mode of the beam [see Fig. 2(b)]. The thermal peaks of higher modes are too small to be detected; therefore we induce external excitation by applying an ac voltage to a nearby parallel electrode, separated from the beam by a gap of width $g=5 \mu \mathrm{m}$. We find three higher modes with frequencies $\nu_{2}=354.4 \mathrm{kHz}, \nu_{3}=529.8 \mathrm{kHz}$, and $\nu_{4}=709.7 \mathrm{kHz}$. The fact that the spectrum obtained is almost equally spaced indicates that $\zeta \ll 1$. Note, however, that drift in the position of the peaks occurring over time prevents us from making a precise estimation of $\zeta$. Based on the uncertainty originated by this drift we place an upper bound of $\zeta<0.015$.

Theoretically, the power of displacement noise near the center of the beam $(x=l / 2)$ around the fundamental frequency for the case $\zeta=0$ is given by

$$
S_{x}(\omega)=\frac{\omega_{0} k_{B} \Theta}{\pi Q m_{\mathrm{eff}}\left[\left(\omega_{0}^{2}-\omega^{2}\right)^{2}+\left(\omega_{0} \omega / Q\right)^{2}\right]},
$$

where $Q$ is the quality factor, $m_{\mathrm{eff}}=\rho A l / 2$ is the effective mass, $\omega=2 \pi \nu$ is the angular frequency, and $\Theta$ is the temperature. Fitting the data in Fig. 2(b) with Eq. (3) yields $Q$ $=1800$. The known parameters of the beam allow determination of the scaling factor translating the signal of the spec- 

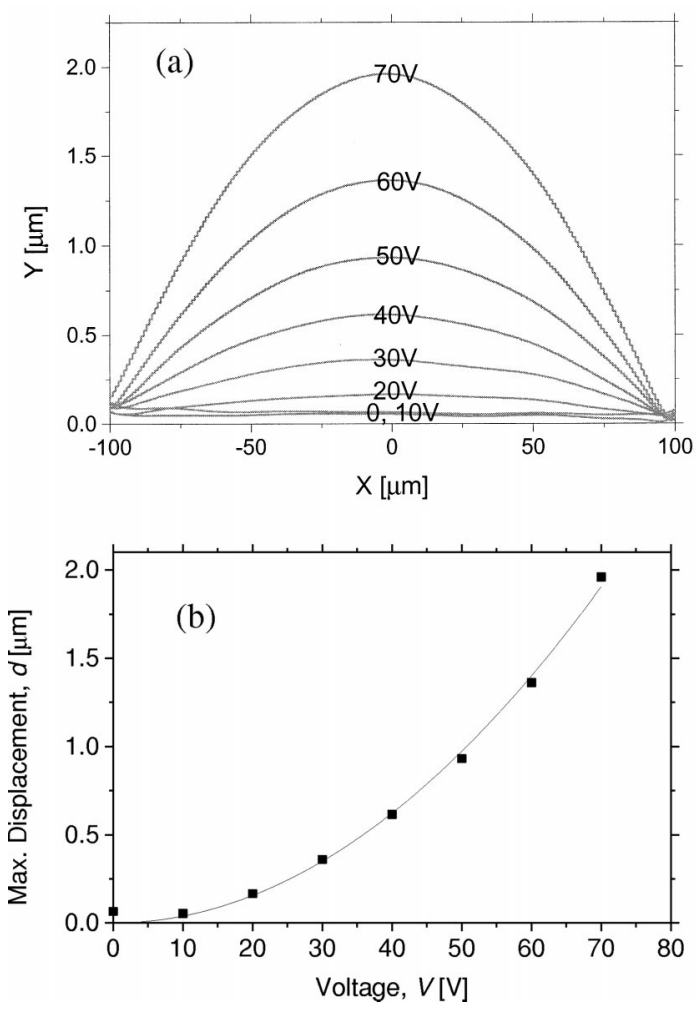

FIG. 3. (a) Deflection of the beam due to application of electrostatic force. (b) Displacement of the center of the beam as a function of applied voltage.

trum analyzer to actual displacement noise. Using this factor and the signal to noise ratio of the data in Fig. 2(b), we find the sensitivity of our displacement detection scheme to be $4 \times 10^{-13} \mathrm{~m} / \mathrm{Hz}^{1 / 2}$. This value can be further enhanced by increasing the current of the electron beam. However, to minimize heating of the device due to electron bombardment we operate at a relatively low current of $100 \mathrm{pA}$. The energy absorbed by the sample depends on the penetration depth of electrons and on the thickness of the Au layer. For an acceleration voltage of $40 \mathrm{kV}$ we estimate the heating power is of order $100 \mathrm{nW} .{ }^{12}$ For thermal conductivity of $300 \mathrm{~W} / \mathrm{mK}$ and the geometry of our device the temperature increase is $\approx 1 \mathrm{~K}$.

To further establish our findings we study the deflection of the beam due to application of a uniform force. For this we apply a dc voltage $V$ between the beam and the nearby electrode. When the deflection is small compared to the distance between the beam and the electrode the force acting on the beam is approximately uniform. The expected deflection is found from the steady state solution of Eq. (1) with $f$ = const:

$$
y(x)=\frac{f l^{2}}{2 T}\left[\frac{1-(2 x / l)^{2}}{4}+\frac{\zeta[\cosh (x / \zeta l)-\cosh (1 / 2 \zeta)]}{\sinh (1 / 2 \zeta)}\right] .
$$

The deflection is observed experimentally by applying voltage $V=0,10, \ldots, 70 \mathrm{~V}$ and imaging the bent beam using SEM. Using image processing we extract the shape of

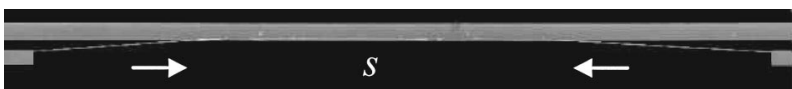

FIG. 4. Adhesion between the beam and a nearby electrode.

the beam in each picture, namely, the experimental value of $y(x)$ [see FIg. 3(a)]. Comparing the calculated $y(x)$ with experimental data using a least squares fit, we determine the parameter $\zeta=0.014 \pm 0.007$, in agreement with the above mentioned estimate of $\zeta$.

The value $\zeta=0.01$ and the other known parameters allow estimating Young's modulus $E=8 \times 10^{10} \mathrm{~N} / \mathrm{m}^{2}$. This value shows reasonable agreement with previous measurements of $E$ in thin films of evaporated gold using different methods. ${ }^{13-15}$

Figure 3(b) shows the maximum displacement of the beam, namely, $y(0)$, as a function of the voltage $V$. As expected, we find that this maximum displacement is proportional to $V^{2}$. Using the value of $T=5.8 \times 10^{-6} \mathrm{~N}$ found from the spectrum measurements we find that $f / V^{2}=4.6$ $\times 10^{-7} \mathrm{~N} / \mathrm{mV}^{2}$.

To study adhesion in our system we bring the beam and the nearby electrode into contact by introducing a pure liquid to the surface of the sample and employing the resultant capillary forces. During drying a thin layer of liquid is formed between the gold surfaces. The pressure inside the drop is lower than the pressure outside if the wetting angle is smaller than $\pi / 2$, resulting in a net attractive force between the surfaces. We employ deionized (DI) water as an adhesive liquid due to its relatively high surface tension ( $\cong 0.07 \mathrm{~N} / \mathrm{m}$ at room temperature).

Figure 4 is a micrograph of the gold beam after drying the DI water from the surface of the sample. The length of the segment that adheres (where the gap is not observable in the SEM) is $s=67.8 \mu \mathrm{m}$. The fact that adhesion between the beam and the nearby electrode persists after drying indicates that the total energy of the adhering system is lower than that of a straight free beam, which is merely metastable.

To estimate the total energy of the system we make two simplifying assumptions: (a) no stiffness, namely, $\zeta=0$ (the measured value $\zeta \simeq 0.01$ justifies this approximation); (b) no finite range interaction between the surfaces (the error due to this approximation is small due to the rapid decay of the interaction as a function of distance). Using the first assumption we find an expression for the elastic energy of the system,

$$
U_{e}=2 g^{2} T /(l-s) .
$$

The second assumption implies that the energy due to the surface-surface interaction is given by

$$
U_{a}=-s t \gamma,
$$

where $\gamma$ is the energy of adhesion per unit area. The condition that the total energy of the system has a minimum implies

$$
\gamma=2 g^{2} T / t(l-s)^{2} .
$$


Using the parameters of our sample we find $\gamma$ $=0.066 \mathrm{~J} / \mathrm{m}^{2}$. A similar value of $0.062 \mathrm{~J} / \mathrm{m}^{2}$ is obtained from another beam with a gap $g=3 \mu \mathrm{m}$.

What is expected theoretically? The Casimir force for small separation is reduced to the so-called nonretarded van der Waals force. ${ }^{10}$ In this regime the separation between the metallic surfaces is small compared to the characteristic wavelength of their absorption spectra, and effects due to finite conductivity are strong. The interaction energy per unit area is given by

$$
U=-A / 12 \pi d^{2}
$$

For the case of Au it was found that Eq. (8) is a good approximation for $d<2 \mathrm{~nm}$ and the Hamaker constant $A$ is given by $A=4.4 \times 10^{-19} \mathrm{~J}$. ${ }^{16}$ This allows estimation of the adhesion energy by $\gamma=A / 12 \pi d_{0}^{2}$, where $d_{0}$ is the effective separation at contact. The nearest neighbor approximation for the case of atomically flat surfaces leads to $d_{0}$ $\approx 0.16 \mathrm{~nm},{ }^{10}$ and theerfore $\gamma \approx 0.4 \mathrm{~J} / \mathrm{m}^{2}$. For the case of metals it was shown that electron exchange interaction (giving rise to the so-called metallic bond) is expected to further enhance $\gamma .{ }^{17}$ The enhancement factor, however, strongly depends on the twist angle between the contacting lattices. Previous measurements of $\gamma$ of metals found values in the range of $0.4-4 \mathrm{~J} / \mathrm{m}^{2}$. $^{10}$

There are two possible explanations for the factor of 6 discrepancy between our results and theory dealing with ideal surfaces. The first is roughness existing on the surfaces in contact. From the measured value of $\gamma$, the calculated value of $A$, and the relation $\gamma=A / 12 \pi d_{0}^{2}$, we find an effective value for the separation between the surfaces within our sample of $d_{0} \approx 0.4 \mathrm{~nm}$. Note that this distance scale for $d_{0}$ is far smaller than can be resolved using SEM or AFM. Another possible cause for the discrepancy might be surface contamination which can strongly modify the adhesion energy even when this is from adsorbed layers only a monolayer thick. ${ }^{10}$
Apart from determining the adhesion energy, a central question is whether we can study the Casimir interaction at finite separation with such stiction experiments. In the immediate vicinity of the region of the beam that is in contact with the electrode, the separation between the beam and electrode is small. This gives rise to a strong Casimir interaction in this location. In principle such attraction can cause additional bending of the beam, allowing thus determination of the magnitude of the attractive force using Eq. (1). To examine this possibility we estimate this additional bending assuming that the attractive interaction is given by Eq. (8) with $A$ $=4.4 \times 10^{-19} \mathrm{~J}$. We assume $\zeta=0$ and solve Eq. (1) using the other known parameters of the beam. We find that the change in the separation between the beam and the electrode becomes comparable to the unperturbed value only when the separation is less than $1 \mathrm{~nm}$. Resolving such a small effect is very difficult with a SEM but might be possible with transmission electron microscope imaging if charging does not become an issue. However, we find that the effect of stiffness on the shape of the beam is much stronger than that due to Casimir attraction. Note, however, that observation of such Casimir induced bending may be easier using a stress-free material with a low Young's modulus and employing a modified geometry.

As demonstrated by the present work, MEMS can provide ideal tools for characterizing stress in thin films as well as for studying adhesion forces. Future experiments with enhanced sensitivity should enable studies of the Casimir force at finite separations.

The authors are grateful to K. Schwab for his assistance in sample fabrication and Y. Buks for image processing of the SEM micrographs. This research was supported by the Rothschild Foundation, the R. A. Millikan Foundation at Caltech, and DARPA MTO/MEMS under Grant No. DABT63-98-1-0012.
${ }^{1}$ H. B. G. Casimir, Proc. K. Ned. Akad. Wet. 51, 793 (1948).

${ }^{2}$ S. K. Lamoreaux, Am. J. Phys. 67, 850 (1999).

${ }^{3}$ S. K. Lamoreaux, Phys. Rev. Lett. 78, 5 (1997); 81, 5475 (1998).

${ }^{4}$ U. Mohideen and A. Roy, Phys. Rev. Lett. 81, 4549 (1998).

${ }^{5}$ J. C. Long, H. W. Chan, and J. C. Price, Nucl. Phys. B 539, 23 (1999).

${ }^{6}$ Niels Tas, Tonny Sonnenberg, Henri Jansen, Rob Legtenberg, and Miko Elwenspoek, J. Micromech. Microeng. 6, 385 (1996).

${ }^{7}$ Roya Maboudian and Roger T. Howe, J. Vac. Sci. Technol. B 15, 1 (1997).

${ }^{8}$ E. Buks and M. L. Roukes, cond-mat/0008096 (unpublished).

${ }^{9}$ F. Michael Serry, Dirk Walliser, and Jordan Maclay, J. Appl. Phys. 84, 2501 (1998).

${ }^{10}$ J. N. Israelachvili, Intermolecular and Surface Forces (Academic
Press, London, 1992).

${ }^{11}$ L. D. Landau and E. M. Lifshitz, Theory of Elasticity (Pergamon, Oxford, 1986).

${ }^{12}$ Ludwig Reimer, Scanning Electron Microscopy (SpringerVerlag, Berlin, 1985).

${ }^{13}$ C. A. Neugebauer, J. Appl. Phys. 31, 1096 (1960).

${ }^{14}$ A. Kinbara, S. Baba, and N. Matuda, Thin Solid Films 141, 229 (1986).

${ }^{15}$ T. P. Weihs, S. Hong, J. C. Bravman, and W. D. Nix, J. Mater. Res. 3, 931 (1988).

${ }^{16}$ G. L. Klimchitskaya, U. Mohideen, and V. M. Mostepanenko, quant-ph/0003093 (unpublished).

${ }^{17}$ J. Ferrante and J. R. Smith, Phys. Rev. B 31, 3427 (1985). 